\title{
Sequential argon-YAG laser iridotomies in dark irides
}

\author{
Tony Ho, Richard Fan
}

\begin{abstract}
Laser iridotomies in dark coloured irides are associated with higher complication and failure rates. A prospective clinical study to evaluate the use of the argon and Nd:YAG laser in sequential combination for iridotomy was carried out on 20 eyes of 13 patients with dark irides. Patent iridotomies were achieved in single treatment sessions for all the eyes. Iridotomy closure was observed in one eye during a mean follow-up period of 14 months (range 6-20 months). Complications from the treatment occurred in five eyes and were minor. Total mean energy used for the argon and Nd:YAG stages respectively were a third of most studies on pure argon and Nd:YAG iridotomies. Sequential argon-YAG laser iridotomy combines most of the advantages of both laser types while avoiding some of their disadvantages. We find it a safe and effective tool for iridotomy in otherwise difficult dark irides.
\end{abstract}

Laser iridotomy is now a well established procedure for the management of angle closure glaucoma and for prophylaxis in fellow eyes. Many ophthalmologists consider the Nd:YAG laser the ideal iridotomy tool especially for blue irides. However the YAG laser does not work as effectively in thick dark brown irides. The higher complication and failure rates in these patients whether by argon or YAG laser are well recognised and documented. ${ }^{1-4}$

Schwartz ${ }^{1}$ using a pulsed argon laser reported $20 \%$ failure to penetrate in brown eyes. Quigley ${ }^{2}$ reported needing two or more sessions to achieve patent argon iridotomies in $58 \%$ of his patients with dark brown irides. Tomey ${ }^{3}$ stated that the higher amount of YAG laser energy required by his patients was attributed to the high prevalence of thick, brown irides among his patients.

Some authors ${ }^{45}$ have documented the

Department of Ophthalmology, Tan Tock Seng General Hospital, Singapore T Ho

R Fan

Correspondence to: Tony Ho, FRCS, FCOphth, Singapore National Eye Centre, 11 Third Hospital Avenue, Singapore 0316.

Accepted for publication 29 November 1991 secondary use of the Nd:YAG laser at separate treatment sessions to salvage an otherwise failed argon iridotomy. We conducted this study to evaluate a method of iridotomy using the argon and $\mathrm{Nd}$ :YAG laser in sequential combination at the same sitting to penetrate the iris.

\section{Materials and methods}

Twenty eyes of 13 patients were treated. All eyes had dark brown irides and were treated by the same investigator. There were 11 Chinese and two Indian patients. Ten eyes had creeping angle closure glaucoma, three had acute angle closure, and seven were contralateral eyes (Table 1). The mean period of follow-up was 14 months (range 6-20 months). The average age of the patient was 62 years (range $45-82$ ).

We used the Coherent Medical 920 Argon laser with a Zeiss slit-lamp delivery system for the first stage and the Cooper Vision 2300 QSwitched Neodymium YAG laser for the second perforation stage.

Prior to the laser therapy all patients had a complete ophthalmic examination. Eyes with very shallow anterior chambers, iritis, or corneal oedema were excluded. Informed consent was obtained and topical pilocarpine $2 \%$ four times daily was administered the day before the procedure. After administration of topical anaesthesia an Abraham lens was inserted. Whenever possible an iris crypt in the mid-periphery was selected as the iridotomy site. The argon laser was used as the first stage laser. Initial argon laser parameters were standardised at (1) spot size $50 \mu \mathrm{m}$, (2) power $700 \mathrm{~mW}$, and (3) duration $0 \cdot 1$ second. We found it important to pre-empt the formation of a black pigment crust as this will absorb the incident laser energy without vaporising and effectively forms a barrier to further penetration. Should charring occur the duration and/or power may need to be shortened and/or reduced. Good penetration was characterised by vapour bubble formation with minimal iris charring. It was not advisable to shoot through a large vapour bubble as accurate focus and visualisation cannot be ascertained. Large vapour bubbles at the iridotomy site can be displaced easily by tapping the Abraham lens with a finger. On the other hand, we found that small vapour bubbles serve as a condensing lens and shooting through these was safe and resulted in good penetrating shots. The inital bore was made to an estimated depth of about two thirds iris thickness with an approximate diameter of 300 to $500 \mu \mathrm{m}$.

The Nd:YAG laser was used as the second stage laser to achieve iris perforation. Energy levels were set between 2 and $5 \mathrm{~mJ}$ depending on the surgeon's estimate of the depth of penetration 
achieved at the end of the first stage. All shots were delivered in single pulse mode.

All patients had their IOP measured at 1 hour postoperatively. Patients with an IOP rise not greater than $10 \mathrm{~mm} \mathrm{Hg}$ above preoperative levels were given prophylactic Diamox (acetazolamide) $250 \mathrm{mg}$ twice daily for the next 2 days. If the IOP rise was greater than $10 \mathrm{~mm} \mathrm{Hg}$ at 1 hour postoperatively the patient was given an immediate dose of $500 \mathrm{mg}$ acetazolamide and monitored for up to 3 hours as it is well documented that most IOP rises occur within the first 3 hours postoperatively. $^{6-8}$ Topical steroids were given for 1 week to all patients after the procedure.

\section{Results}

We were able to achieve patent iridotomies in all 20 eyes at the same sitting. For the first argon laser stage the mean number of shots needed to achieve the initial bore was 55 (SD 26, range 15140; Tables 2 and 3). The mean power used was $810 \mathrm{~mW}$ (SD 181, range 600-1100; Tables 2 and 3 ). It was often necessary to vary the duration of the shots during the procedure to achieve penetration and to avoid charring the iris. The duration of the argon laser shots ranged from 0.01 second to 0.1 second (Table 2). For the second perforation stage, which was done using the Nd:YAG laser, the mean number of shots needed was 4 (SD 2, range 1-6; Tables 2 and 3). Energy settings usually started off at $2 \mathrm{~mJ}$ depending on the surgeon's estimate of the depth of iris penetration achieved at the end of the first stage. The mean energy setting was $4 \mathrm{~mJ}$ (SD $1 \cdot 2$, range $2-5$ ).

The mean total energy delivered per eye by the argon and Nd:YAG lasers respectively were $3.6 \mathrm{~J}$ and $9.4 \mathrm{~mJ}$.

Table 2 Sequential argon-YAG iridotomy-laser parameters

\begin{tabular}{|c|c|c|c|c|c|c|}
\hline Patient no & $\begin{array}{l}\text { Glaucoma } \\
\text { type }\end{array}$ & $\begin{array}{l}\text { No of } \\
\text { argon } \\
\text { shots }\end{array}$ & $\begin{array}{l}\text { Average } \\
\text { power } \\
(m W)\end{array}$ & $\begin{array}{l}\text { Average } \\
\text { duration } \\
\text { (seconds) }\end{array}$ & $\begin{array}{l}\text { No of } \\
\text { YAG } \\
\text { shots }\end{array}$ & $\begin{array}{l}\text { Energy } \\
\text { setting } \\
(m \mathcal{f})\end{array}$ \\
\hline 1 & CAC & 50 & 900 & 0.05 & \multirow{17}{*}{$\begin{array}{l}2 \\
1 \\
4 \\
1 \\
4 \\
2\end{array}$} & \multirow{17}{*}{$\begin{array}{l}2 \\
2 \cdot 4 \\
3 \\
2 \cdot 4 \\
4 \\
4 \\
5 \\
2 \cdot 5 \\
3 \\
2 \cdot 5 \\
2 \cdot 4 \\
2 \\
2 \cdot 5 \\
4 \\
2 \\
5 \\
5 \\
5 \\
5 \\
3 \\
5 \\
3 \\
5\end{array}$} \\
\hline & FE & 50 & 1100 & 0.05 & & \\
\hline 2 & AAC & 50 & 700 & 0.03 & & \\
\hline & FE & 50 & 600 & 0.02 & & \\
\hline 3 & AAC & 34 & 1100 & $0 \cdot 1$ & & \\
\hline & FE & 38 & 1100 & $0 \cdot 1$ & & \\
\hline $\begin{array}{l}4 \\
5\end{array}$ & $\begin{array}{l}\text { AAC } \\
\text { FE }\end{array}$ & $\begin{array}{l}50 \\
50\end{array}$ & $\begin{array}{l}700 \\
700\end{array}$ & $\begin{array}{l}0.1 \\
0.1\end{array}$ & & \\
\hline & FF & 40 & 700 & 0.1 & & \\
\hline 7 & FE & 15 & 1000 & 0.1 & & \\
\hline 8 & $\begin{array}{l}\text { CAC } \\
\text { CAC }\end{array}$ & $\begin{array}{r}82 \\
140\end{array}$ & $\begin{array}{r}1000 \\
700\end{array}$ & $\begin{array}{l}0.01 \\
0.01\end{array}$ & & \\
\hline 9 & AAC & 70 & 700 & $0 \cdot 1$ & & \\
\hline & CAC & 70 & 700 & $0 \cdot 1$ & & \\
\hline 10 & CAC & 45 & 600 & 0.1 & & \\
\hline & CAC & 50 & 700 & $0 \cdot 1$ & & \\
\hline 11 & CAC & 18 & 1100 & $0 \cdot 1$ & & \\
\hline 12 & CAC & 50 & 700 & $0 \cdot 1$ & & \\
\hline 13 & $\begin{array}{l}\text { CAC } \\
\text { FE }\end{array}$ & $\begin{array}{l}80 \\
62\end{array}$ & $\begin{array}{l}600 \\
800\end{array}$ & $\begin{array}{l}0.1 \\
0.1\end{array}$ & & \\
\hline
\end{tabular}

Table 3 Mean laser parameters

\begin{tabular}{lcc}
\hline lst stage & Mean argon parameters $(S D)$ & \\
Power $(\mathrm{mW})$ & $810(181)$ & (range 600-1100) \\
Exposure (seconds) & $0 \cdot 08(0 \cdot 03)$ & (range 0.01-0.1) \\
No of shots & $55 \cdot 0(26 \cdot 0)$ & (range 15-140) \\
Mean total energy delivered per eye $(\mathrm{J})$ & $3 \cdot 6$ & \\
2nd stage & $M e a n$ ND:YAG laser parameters $(S D)$ \\
Energy setting (per shot, $\mathrm{mJ}$ ) & $4 \cdot 0(1 \cdot 2)$ & \\
No of shots (single mode) & $4 \cdot 0(2 \cdot 0)$ & (range 1-9) \\
Mean total energy delivered per eye (mJ) & $9 \cdot 4$ & \\
\hline
\end{tabular}

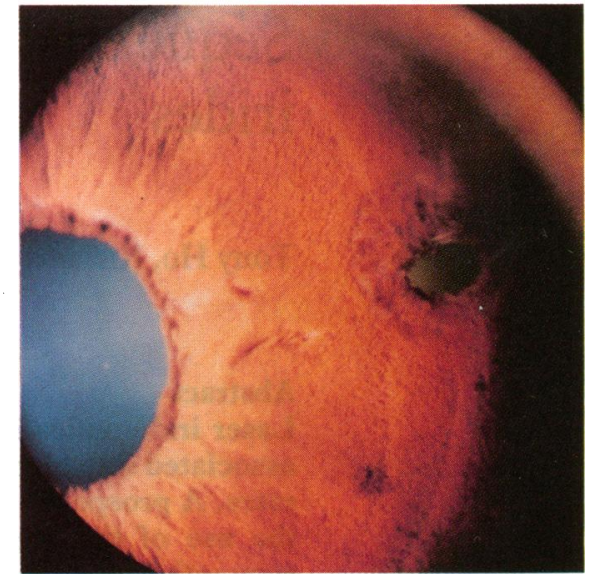

Figure 1 Typical sequential argon-YAG iridotomy. Large, round iridotomy with sharp margins.

Microhyphaema was seen in two eyes. Focal lens opacity was noted in one eye. Focal corneal endothelial opacity was seen in two eyes. Immediately following the iridotomy all the eyes had various degrees of pigment dispersion and free floating debris. However no clinically significant inflammation was present by the end of the first week. Five of the eyes had intraocular pressure rises over $10 \mathrm{~mm} \mathrm{Hg}$ within the first hour. However they responded well to acetazolamide treatment. Iridotomy closure was observed in one eye during a mean follow-up period of 14 months (range 6-20 months).

\section{Discussion}

Certain features of the argon laser makes it an effective iridotomy tool in dark coloured irides. (1) The incident laser energy is well absorbed and penetration can therefore be achieved at relatively low thermal settings, (2) hyphaema, which is a common occurrence with YAG iridotomies is uncommon, and (3) pigment dispersion is minimal compared with YAG iridotomies.

However the high energy absorption often causes significant charred pigment crust formation which acts as a barrier to further penetration. This, together with the thicker iris,

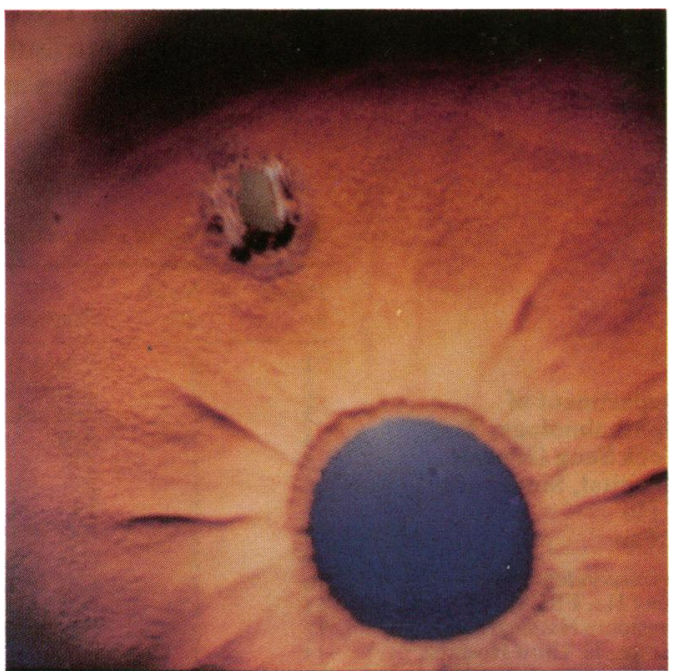

Figure 2 Another example of iridotomy achieved with this method. 


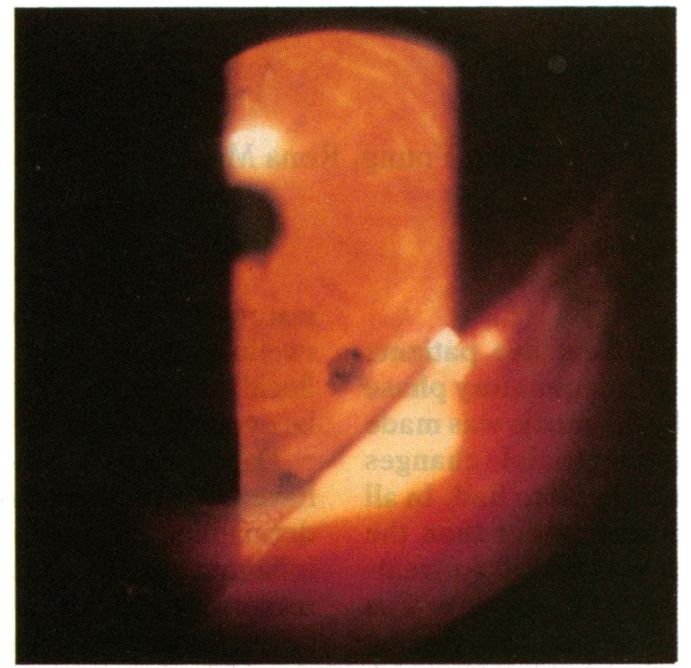

Figure 3 Patient at end of first argon laser stage.

means more shots are often needed to create a patent iridotomy. Indeed Ritch' has reported using 300 to 400 shots with argon laser settings at $1 \mathrm{~W}$ power, 0.02 second duration, and $50 \mu \mathrm{m}$ spot size in patients with dark brown irides. This is of concern as central endothelial cell loss due to the indirect thermal effects of the laser has been documented. ${ }^{6}$ In our study we found the mean total energy delivered per eye by the argon and YAG respectively is only a third of the corresponding values reported for pure argon and YAG iridotomies. $^{6-8}$ This should have a significant bearing on complications resulting from either direct or indirect thermal damage to the eye.

The iridotomies created were typically large, round, and with sharp margins (Figs 1 and 2). This is in contrast to the slit opening of YAG iridotomy and the argon iridotomy where the hole is smaller with charred edges.

Complications resulting from the treatment were minor. Microhyphaema was seen in two eyes $(10 \%)$. This is in contrast to pure YAG iridotomies where about $40 \%$ are complicated by bleeding. ${ }^{6-8}$ This advantage is probably conferred

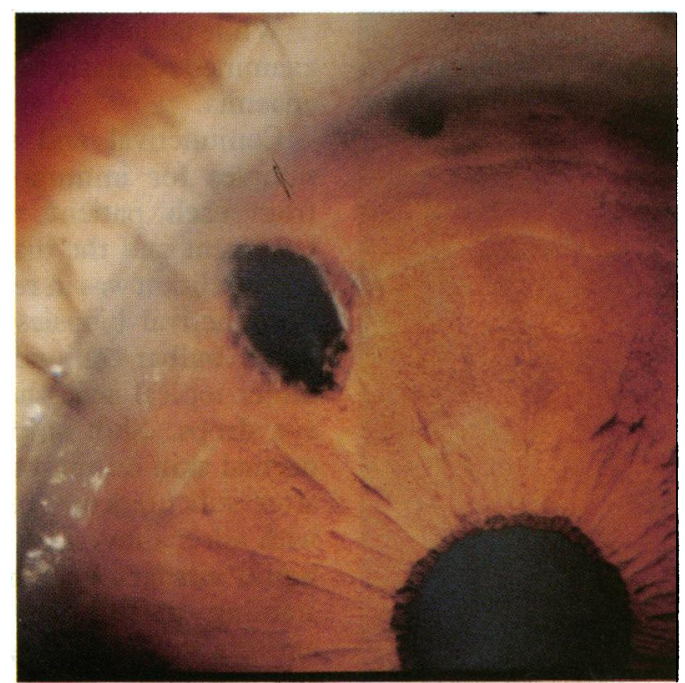

Figure 4 Same patient as Figure 3 at 10 months follow-up. In this patient the iridotomy demonstrated spontaneous enlargement to twice its original size. by the use of the argon laser as the first stage laser.

One of the major advantages of YAG iridotomy is that it is rarely associated with focal lens damage. This is because the zone of optical breakdown and plasma formation following YAG laser shots extends not distally but proximally towards the laser source, a phenomenon termed the 'plasma shield effect'. On the other hand the incidence of focal lens damage in argon iridotomy has been reported to be between 35 and $53 \% .^{6-8}$ Our study showed a low incidence possibly due to the use of the YAG laser as the second stage laser.

Focal corneal endothelial opacity was seen in two eyes $(10 \%)$ and this rate is similar to that reported by most studies on pure argon and YAG iridotomies. ${ }^{6-8}$

Closure rates of $21-31 \%$ have been reported in pure argon laser iridotomy whereas it is well established that YAG laser iridotomy appears to show no tendency to close in eyes uncomplicated by rubeosis or iritis although the exact mechanism remains unclear. ${ }^{78}$ Iridotomy closure was noted in only one eye during a mean follow-up period of 14 months (range 6-20 months). Could the use of the YAG as the second stage laser be the reason for these low closure rates?

An interesting phenomenon of spontaneous progressive iridotomy enlargement was observed in some eyes. In one particular patient the iridotomy enlarged to almost twice the original iridotomy size over a 10 month follow-up period (Figs 3 and 4). It is our clinical impression that this phenomenon occurred in about a third of the eyes, albeit to a much lesser degree.

We observed two further advantages from this procedure. (1) Macula injury from inadvertent argon laser shots is very unlikely with this method and therefore one is not restricted to using only the nasal side for the iridotomy site. (2) The hazardous and time consuming chipping and enlargement phase necessary in argon iridotomy is not necessary.

\section{Conclusion}

This study suggests that sequential argon-YAG iridotomy is a safe and effective procedure and combines the advantages of both laser procedures while avoiding most of their disadvantages. It could well be the ideal iridotomy tool for dark coloured irides.

1 Schwartz LW, Rodrigues MM, Spaeth GL, Streeten B, Douglas C. Argon laser iridotomy in the treatment of patients with primary angle-closure or pupillary block glaucoma: a clinicopathologic study. Am f Ophthalmol 1978; 85: 294-309.

2 Quigley HA. Long term follow up of laser iridotomy. $A m \boldsymbol{F}$ Ophthalmol 1981; 88: 218-24.

3 Tomey KF, Traverso CE, Shammas IV. Nd:YAG laser iridotomy in the treatment and prevention of angle closure glaucoma. Arch Ophthalmol 1987; 105: 476-81.

4 Naveh-Floman, Blumenthal M. A modified technique for serial use of argon and Nd:YAG lasers in laser iridotomy. $A m \mathcal{F}$ Ophthalmol 1985; 100: 485 .

5 Robin AL, Pollack IP. Q-switched Nd:YAG laser iridotomy in patients in whom the argon laser fails. Arch Ophthalmol 1986; 104: 531-5.

6 Robin AL, Pollack IP. A comparison of Nd:YAG and argon laser iridotomies. Am $\mathcal{F}$ Ophthalmol 1984; 91: $1011-6$.

7 Del Priore LV, Robin AL, Pollack IP. Nd:YAG and argon laser iridotomy - long term follow-up in a prospective, randomized clinical trial. Am f Ophthalmol 1988; 95: 1207-11.

8 Moster MR, Schwartz LW, Spaeth GL, Wilson RP, McAllister JA, Poryzees EM. A controlled study comparing argon and Nd:YAG iridectomy. Am 7 Ophthalmol 1986; 93: 20-4.

9 Ritch R. Techniques of argon laser iridectomy and iridoplasty. Palo Alto, CA: Coherent Medical, 1983. 